\title{
Exclusive endoscopic transcanal transpromontorial approach: a new perspective for internal auditory canal vestibular schwannoma treatment
}

\author{
Daniele Marchioni, MD, ${ }^{1}$ Matteo Alicandri-Ciufelli, MD, FEBORL-HNS, ${ }^{2,3}$ Alessia Rubini, MD, ${ }^{1}$ \\ Babara Masotto, ${ }^{4}$ Giacomo Pavesi, MD, ${ }^{3}$ and Livio Presutti, MD ${ }^{2}$ \\ Departments of ${ }^{1}$ Otolaryngology-Head and Neck Surgery and ${ }^{4}$ Neurosurgery, University Hospital of Verona, Verona; \\ 2Department of Otolaryngology-Head and Neck Surgery, University Hospital of Modena, Modena; and ${ }^{3}$ Department of \\ Neurosurgery, New Civil Hospital Sant'Agostino-Estense, Baggiovara, Italy
}

\begin{abstract}
OBJECTIVE The aim of this study was to describe the first case series in which an exclusive endoscopic transcanal transpromontorial approach (EETTA) was used to treat small vestibular schwannomas (VSs) and meningiomas of the internal auditory canal (IAC).
\end{abstract}

METHODS The authors performed a retrospective review of patients who had undergone surgery using an EETTA to the IAC at 2 university tertiary care referral centers during the period from November 2011 to January 2015.

RESULTS Ten patients underwent surgery via an EETTA for the treatment of VS in the IAC at the University Hospital of Modena or the University Hospital of Verona. The patients had Koos Grade I or II tumors and American Academy of Otolaryngology-Head and Neck Surgery (AAO-HNS) Class D hearing status preoperatively. Gross-total resection was achieved in all patients. No major complications such as cerebrospinal fluid leakage or hemorrhage were reported. In 7 of $10(70 \%)$ patients, facial nerve function was normal immediately after surgery (Rough Grading System [RGS] Grade I) Two patients presented with a transitory facial palsy immediately after surgery (RGS Grade II-III) but experienced complete recovery during the follow-up period. The mean follow-up was 10 months.

CONCLUSIONS The EETTA proved to be successful for the removal of VS or meningioma involving the cochlea, fundus, and IAC, with possible lower complication rates and less invasive procedures than those for traditional microscopic approaches. The potential for the extensive and routine use of this approach in lateral and posterior skull base surgery will depend on the development of technology and surgical refinements and on the diffusion of skull base endoscopic skills among the otolaryngological and neurosurgical communities.

http://thejns.org/doi/abs/10.3171/2015.11.JNS15952

KEY WORDS inner ear; internal auditory canal; vestibular schwannoma; transcanal approach; endoscopic ear surgery; surgical technique

$\mathrm{S}$ URGICAL approaches to treat vestibular schwannoma (VS) are widely known and extensively recorded, with the most popular being the retrosigmoid, transmastoid-translabyrinthine, and middle cranial fossa approaches. Clinical indications, advantages, disadvantages, and risks for mortality and morbidity have been carefully described. ${ }^{2}$ A common factor among these methods is their indirect approach to the inner ear; the retrosigmoid and translabyrinthine methods are posterior approaches, while the middle cranial fossa method is a superior approach. To access the internal auditory canal (IAC) and cerebellopontine angle (CPA), all approaches require wide external incisions and a variable degree of bone removal. ${ }^{2}$

The first endoscopic technique for IAC surgery was introduced in combination with the retrosigmoid approach: ${ }^{4}$ after removal of the CPA portion of the neoplasm, the intracanalicular extension was removed under endoscopic control, trying to avoid extensive drilling of the posterior

ABBREVIATIONS AAO-HNS = American Academy of Otolaryngology-Head and Neck Surgery; CPA = cerebellopontine angle; CS = cochlear schwannoma; CSF = cerebrospinal fluid; EAC = external auditory canal; EETTA = exclusive endoscopic transcanal transpromontorial approach; IAC = internal auditory canal; ICU = intensive care unit; RGS = Rough Grading System; VS = vestibular schwannoma.

SUBMITTED April 28, 2015. ACCEPTED November 18, 2015.

INCLUDE WHEN CITING Published online March 11, 2016; DOI: 10.3171/2015.11.JNS15952. 
aspect of the petrous bone. Endoscopic techniques for the treatment of middle ear pathologies have been gradually introduced since the 1990s. ${ }^{13}$ Endoscopy was first used primarily for the visualization of hidden areas such as the posterior epitympanum during classic microscopic tympanoplasties. ${ }^{9}$ Gradually, endoscopy became widely used in surgery, replacing the microscope as the primary surgical tool during middle ear operations. ${ }^{6,11}$ At present, endoscopic surgery is mostly used for the treatment of middle ear cholesteatoma, but as the technique has evolved, advantages for its use in lateral skull base surgery have been realized as well. ${ }^{7,10}$ During recent years, we have progressively noticed that the internal ear and all of the temporal bone can be accessed in an endoscope-assisted fashion or even with an exclusive endoscopic approach. The development of these endoscopic techniques required several cadaveric dissections to better understand the anatomy and to define appropriate instruments for this purpose. ${ }^{5}$ Through these dissections, advances were made in the exploration of the internal ear, from the labyrinth to the IAC, until an appropriate procedure was recorded and ready to be applied clinically.

In March 2012, an exclusive endoscopic approach to the IAC was described for the first time and used to remove a cochlear schwannoma (CS). The operation involved a direct transcochlear approach from lateral to medial and from the external auditory canal (EAC) to the IAC, without any external incision. ${ }^{8}$ Our team has detailed other lateral skull base applications during the last 2 years. ${ }^{7,10}$ Here we describe the first case series of patients with intracanalicular VS who had been treated by an exclusive endoscopic transcanal transpromontorial approach (EETTA). Since the management of intracanalicular VS is complex and strongly debated, ${ }^{12}$ this therapeutic option for appropriate and select patients could modify classic concepts in the management of this pathology.

\section{Methods}

From November 2011 to January 2015, 10 patients underwent surgery using an EETTA to the IAC in the otolaryngology department of the University Hospital of Modena or the University Hospital of Verona. Indications for the use of EETTA included 1) a growing mass consistent with VS and detected on MRI, 2) Koos Grade I (mass located in the IAC) or Koos Grade II (IAC involvement and limited extension to the CPA) tumors (Fig. 1), and 3) American Academy of Otolaryngology-Head and Neck Surgery (AAO-HNS) Class D hearing status (from severe to profound hearing loss).

All surgical procedures were performed by 2 senior otolaryngologists (D.M. and L.P.). A cooperating neurosurgeon was also present during surgery. A retrospective chart review of patients who had undergone surgery using the EETTA was performed between February and March 2015. All useful data were collected and reported in the present study.

\section{Surgical Technique}

The patient was placed in a supine position, with the head slightly extended and rotated contralaterally, as in

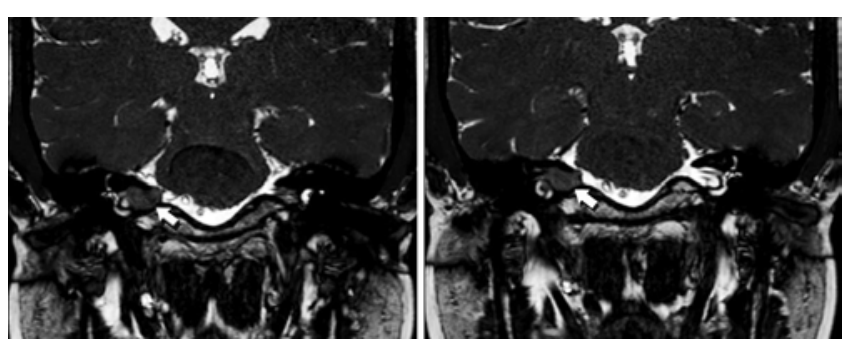

FIG. 1. Coronal T2-weighted MR images showing a typical indication for EETTA: a VS of the IAC without extension to the CPA. White arrows show the medial limit of the VS in the IAC meatus.

traditional endoscopic middle ear surgery. Intraoperative facial nerve monitoring was used for all patients. A 4-mmdiameter, 15-cm-long, and $0^{\circ}$ angle endoscope (Karl Storz) and a 3-chip high-resolution monitor and camera system (AIDA, Karl Storz) were used for all surgeries. The endoscope was held in the left hand and the operative instruments in the right hand. The monitor was positioned in front of the surgeon. All operations were recorded and stored on a hard disk for documentation and further analyses.

No external incisions were made. The procedure was performed using the natural orifice of the external auditory meatus exclusively (Fig. 2A and Video 1).

VIDEO 1. Exclusive endoscopic transcanal transpromontorial approach in the right ear: main steps. Copyright Matteo AlicandriCiufelli. Published with permission. Click here to view.

The endoscope was inserted into the EAC in a fashion similar to that used in a traditional endoscopic ear surgery, ${ }^{6,7}$ then, a circumferential incision of the external ear canal skin was made approximately $3 \mathrm{~cm}$ from the tympanic anulus. The tympanic membrane and EAC skin were removed en bloc, exposing the tympanic cavity and the whole medial aspect of the tympanic cavity (Fig. 2B). Circumferential drilling of the EAC was performed to further increase the view and to facilitate the maneuvering of surgical instruments into the canal (Fig. 3A). The incus and malleus were removed to allow a wide exposition of the whole tympanic tract of the facial nerve (Figs. 2C and 3B) until the geniculate ganglion was seen, locating it between the cochleariform process and the cog. The jugular bulb and carotid artery were detected in the tympanic cavity by drilling the protympanic and hypotympanic areas, which represented the inferior and anterior limits of this approach, respectively (Fig. 3C). The mastoid portion of the facial nerve was detected by drilling the posterior aspect of the EAC and the posterior portion of the bony anulus, which represented the posterior limit of the dissection (Fig. $3 \mathrm{C})$. The stapes was removed from the oval window to expose the vestibule. The inferior lip of the oval window was gently removed with a microcurette, bur, or Piezosurgery instrument (Mectron) to enlarge the opening and expose the saccular fossa in the medial aspect of the vestibule. The spherical recess was also identified in the saccular fossa, appearing like a thin cribriform plate separating the vestibule from the fundus of the IAC and representing the site of medial termination of the inferior vestibular nerve fibers. The promontory was then drilled to expose the basal, middle, and apical turns of the cochlea (Figs. $2 \mathrm{C}$ and $3 \mathrm{C}$ ). 


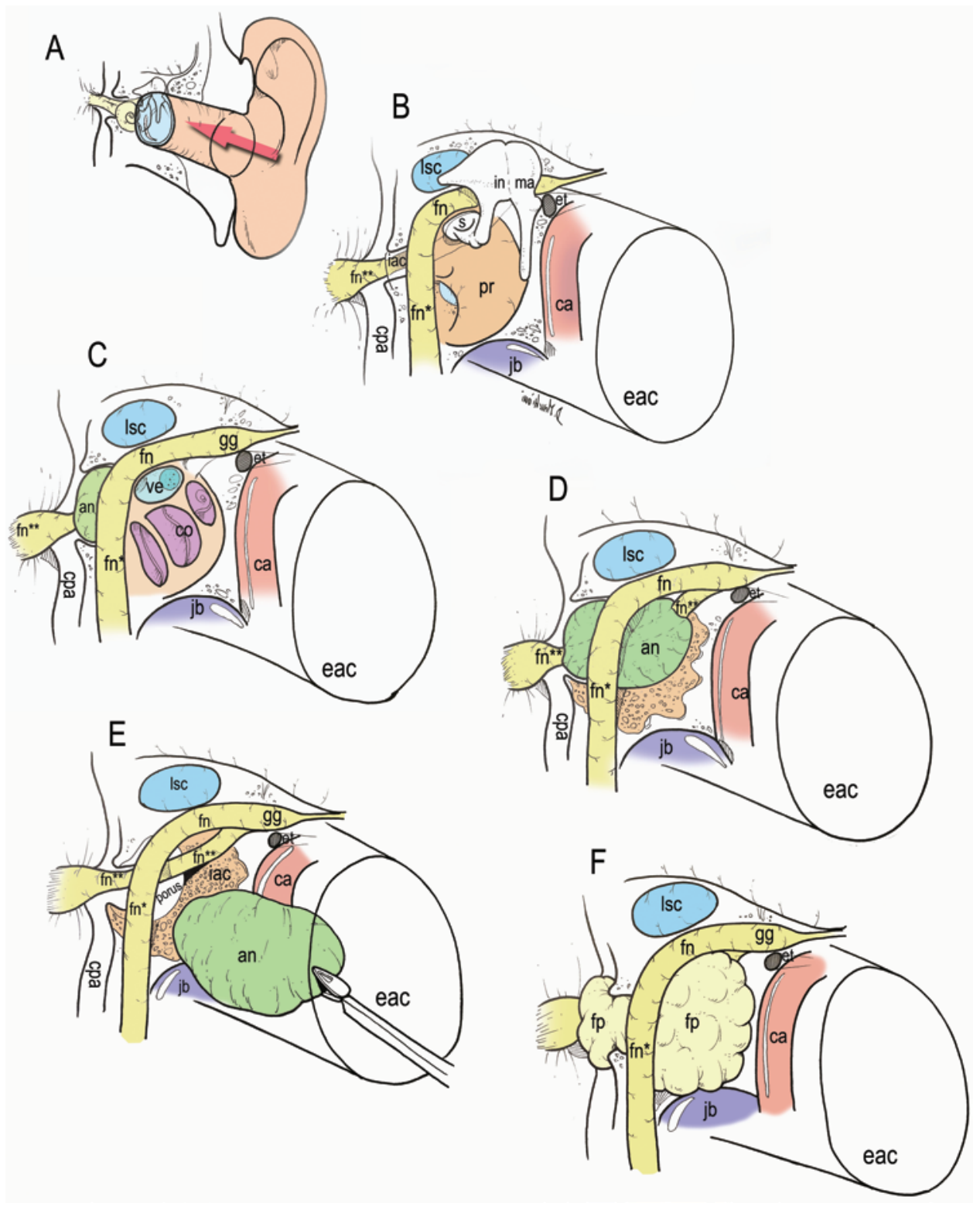

FIG. 2. Schematic of the approach. A: The approach was performed transcanally without any external incision. B: Anatomy and landmarks before the operation. C: After drilling of the cochlea and removal of the ossicles. D: Visualization of the schwannoma after drilling of the IAC. E: Removal of the schwannoma. F: Obliteration of the IAC by a fat pad at the end of the operation. an = acoustic neuroma (VS); $c a$ = carotid artery; et = eustachian tube; $\mathrm{fn}=$ facial nerve (tympanic tract); $\mathrm{fn}^{*}=$ facial nerve (mastoid tract); $f^{* *}=$ facial nerve (IAC segment); $f p=$ fat pad; $g g=$ geniculate ganglion; in = incus; $j b=$ jugular bulb; Isc = lateral semicircular canal; $\mathrm{ma}=$ malleus; $\mathrm{pr}=$ promontory; $\mathrm{s}=$ stapes; ve = vestibule. Copyright Daniele Marchioni. Published with permission. Figure is available in color online only.

After completing these steps, we knew the position of the labyrinthine tract of the facial nerve since all landmarks were exposed; an imaginary line passing from the geniculate ganglion to the spherical recess just above the apical turn of the cochlea, in fact, showed where the facial nerve runs into the inner ear. Next, drilling was performed around the inferior aspect of the vestibule until the fundus of the IAC was opened, exposing the most lateral portion of the pathology. The IAC was increasingly exposed by further drilling around the inferior aspect of the vestibule; the limits of the dissection were the tympanic tract of the facial nerve superiorly, the internal carotid artery anteriorly, the jugular bulb inferiorly, and the mastoid portion of the facial nerve posteriorly. The drilling was performed until the lateral aspect of the IAC dura was completely exposed (Figs. 2D and 3D). We then drilled in a circumferential manner under the vestibule and around the IAC in a horseshoe shape, deepening from lateral to medial to uncover the anterior, inferior, and posterior borders of the IAC (Fig. 4 left) until reaching the reflection of the dura from the IAC meatus to the medial surface of the petrous bone (Fig. 4 right); this represented the deepest landmark 

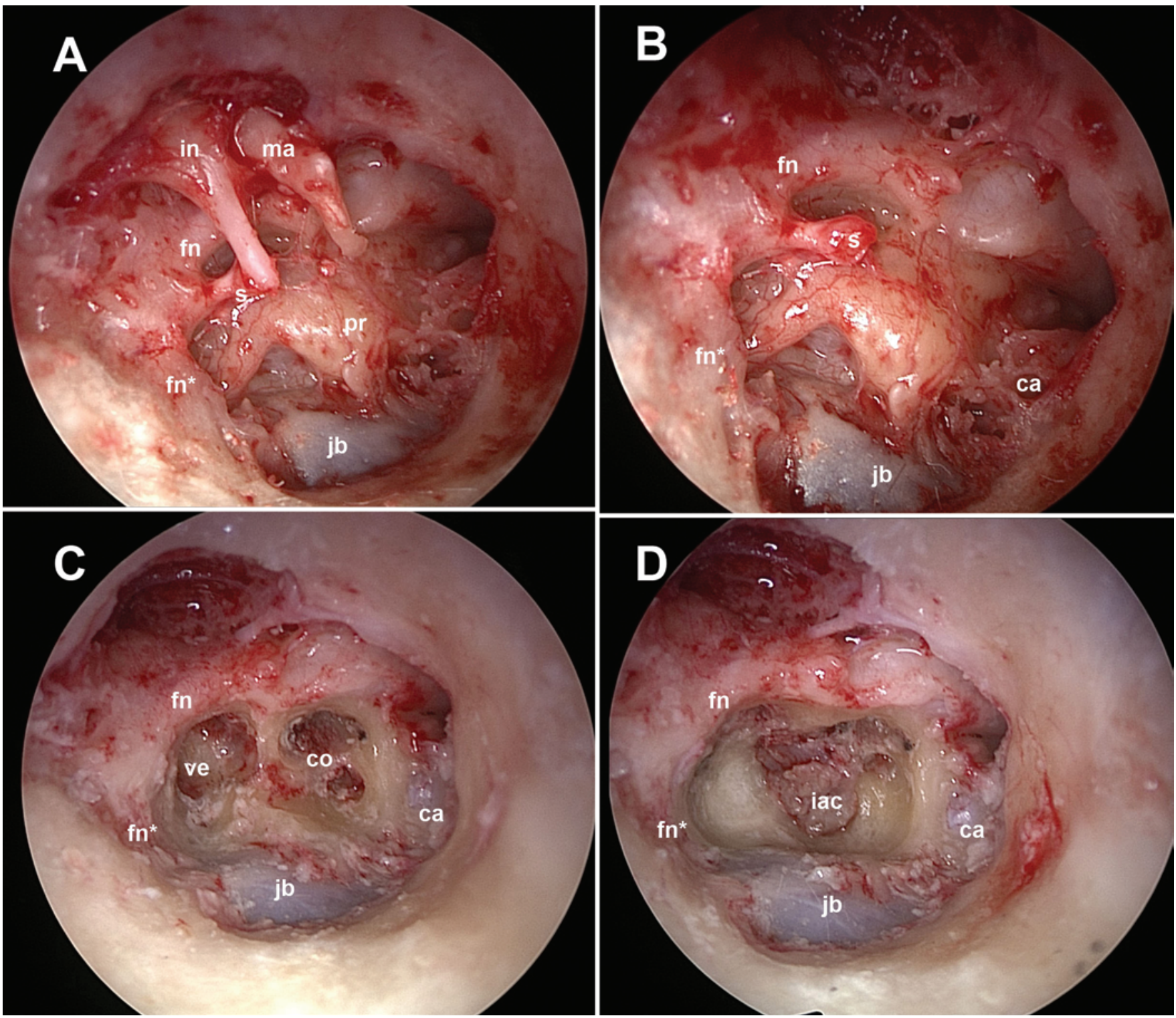

FIG. 3. Photographs of EETTA in the right ear. A: The EAC was widely drilled to expose the tympanic cavity. The mastoid tract of the facial nerve and the jugular bulb were exposed. B: The incus and malleus were removed, and the carotid artery was exposed. C: The stapes was removed to detect the vestibule, and the promontory was removed to expose the turns of the cochlea. D: Additional drilling between the vestibule and the cochlea was performed to expose the IAC. co = cochlea. See Fig. 2 for additional abbreviations. Figure is available in color online only.

of the approach. An incision of the dura was made along the IAC to expose the tumor in the canal. When the mass occupied the entire IAC, central debulking was performed to improve management of the tumor, allowing cerebrospinal fluid (CSF) to drain from the CPA and facilitating visualization of the facial nerve into the IAC. When the facial nerve was detected, the dissection of the tumor from the facial nerve proceeded gently inside the IAC (Figs. 2E and $5 \mathrm{~A})$. The VS was removed from the meatus to the fundus of the IAC, preserving the facial nerve; during those steps, a second surgeon helped the first in some cases by suctioning $\mathrm{CSF}$ and blood, easing the dissection. The tumor was dissected "piecemeal" until a radical removal was achieved.

When extension to the CPA was found, an extended ap- proach was required, and additional bone was drilled to enlarge the opening of the IAC meatus to allow management of the residual tumor lying in the CPA. This drilling was performed by following the acoustic-facial bundle toward the entry zone (Fig. 5B). After complete removal of the tumor, an endoscopic check of the surgical field was made to look for possible residual disease (Fig. 5C).

A fat pad harvested from the abdomen was placed between the inner and middle ear to close the defect (Figs. $2 \mathrm{~F}$ and $5 \mathrm{D}$ ). Fibrin glue was placed over the fat, and a closure of the EAC skin was made. In some patients (mainly the first patients of the case series), a cartilage graft was placed to close the EAC at the level of the bony anulus and to replace the EAC skin. 

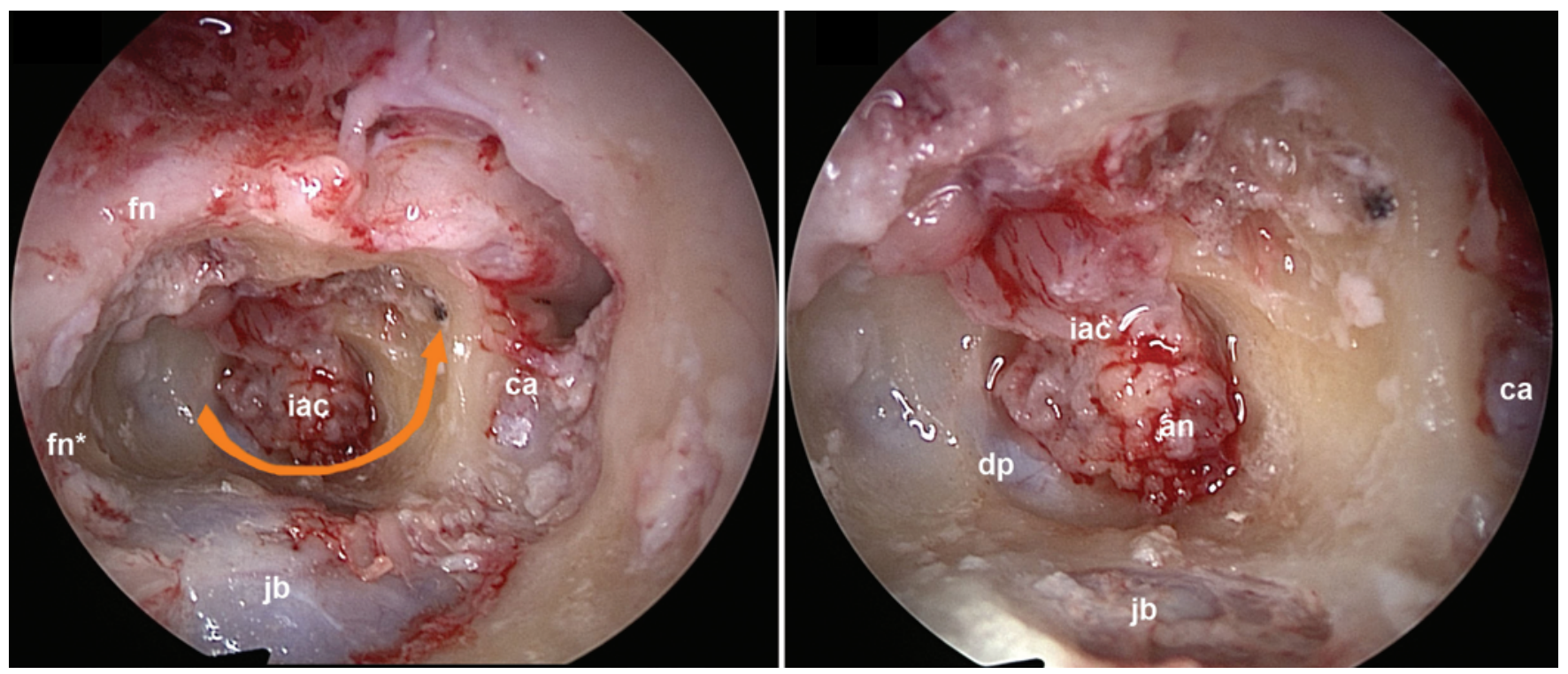

FIG. 4. Photographs of EETTA in the right ear. Left: The IAC was skeletonized by drilling in a circumferential way under the vestibule and around the IAC in a horseshoe shape (orange arrow) from lateral to medial, detecting and uncovering the anterior, inferior, and posterior borders of the IAC. Right: The dissection of the IAC was performed until the reflection of the dura from the porus to the medial surface of the temporal bone into the CPA was reached. $d p=$ reflection of the dura from the porus. See Fig. 2 for additional abbreviations. Figure is available in color online only.

\section{Results}

Ten patients were included in the present study: 4 male and 6 female. The median age was 49 years. The mean follow-up period was 10 months (Table 1).

\section{Surgery}

In all patients, it was possible to remove the VS mass via an EETTA and anatomically preserve the facial nerve. No intraoperative complications were observed in the present series. The average surgery duration was $192 \mathrm{~min}-$ utes. A gross-total resection was achieved in all patients. As expected, all patients had postoperative anacusis on the surgical side as a result of cochlea removal, as occurs in the translabyrinthine approach.

Histologically we identified 9 cases of VS ( 1 of these lesions also extended inside the cochlea, so it was clinically and radiologically classified as a CS) and 1 meningioma.

\section{Postoperative Care}

Intensive care unit (ICU) monitoring and observation was not required for any of the patients, and all patients were immediately extubated after the surgery. No complications were observed on the postoperative CT performed 3-5 hours after surgery. In the postoperative period, a pressure dressing was not used and only a light dressing was applied to cover the auricle and the EAC. All patients maintained bed rest and immobilization in a supine position for 2 days after the surgery. No CSF leaks were verified, and a lumbar drain was not used in any of the patients in the present case series. The average hospital stay was 7.1 days (range 5-10 days), with the first patients staying the longest because of a more cautious attitude at the beginning of our experience.

\section{Facial Nerve Outcome}

In 7 of the 10 (70\%) patients, facial nerve function was normal immediately after the operation (Rough Grading System [RGS] Grade I). ${ }^{1}$ Two patients presented with a transitory facial palsy immediately after surgery (RGS Grade II-III) but experienced complete recovery during the follow-up period. In 3 of the $10(30 \%)$ patients, a facial palsy in the immediate postoperative period was noted (RGS Grade III-IV) and an improvement in facial nerve function was observed (RGS Grade II-III) during the hospital stay.

\section{Discussion}

Management of VS in the IAC is still debated. The IAC is a difficult-to-access anatomical region regardless of the approach chosen. Accessing this region by the retrosigmoid approach requires a craniotomy and extensive drilling of the posterior aspect of the petrous bone to fully expose the IAC. In most cases, the use of an endoscope inside the CPA is required to visualize the fundus of the IAC. Accessing this region by the translabyrinthine approach requires a subtotal petrosectomy to identify the IAC and properly skeletonize it. The middle cranial fossa approach guarantees less bone drilling to expose the petrous bone, but requires a wide craniotomy and temporal lobe retraction. Regardless of the approach chosen, the surgery for VS is traditionally considered very delicate overall. Morbidity can be high, including intraoperative and postoperative complications. In addition, postoperative facial nerve function is critical for psychological issues and quality of life for the patient. For these reasons, the general attitude toward the management of VS in the IAC encourages a wait-and-scan strategy for most patients to evaluate the 

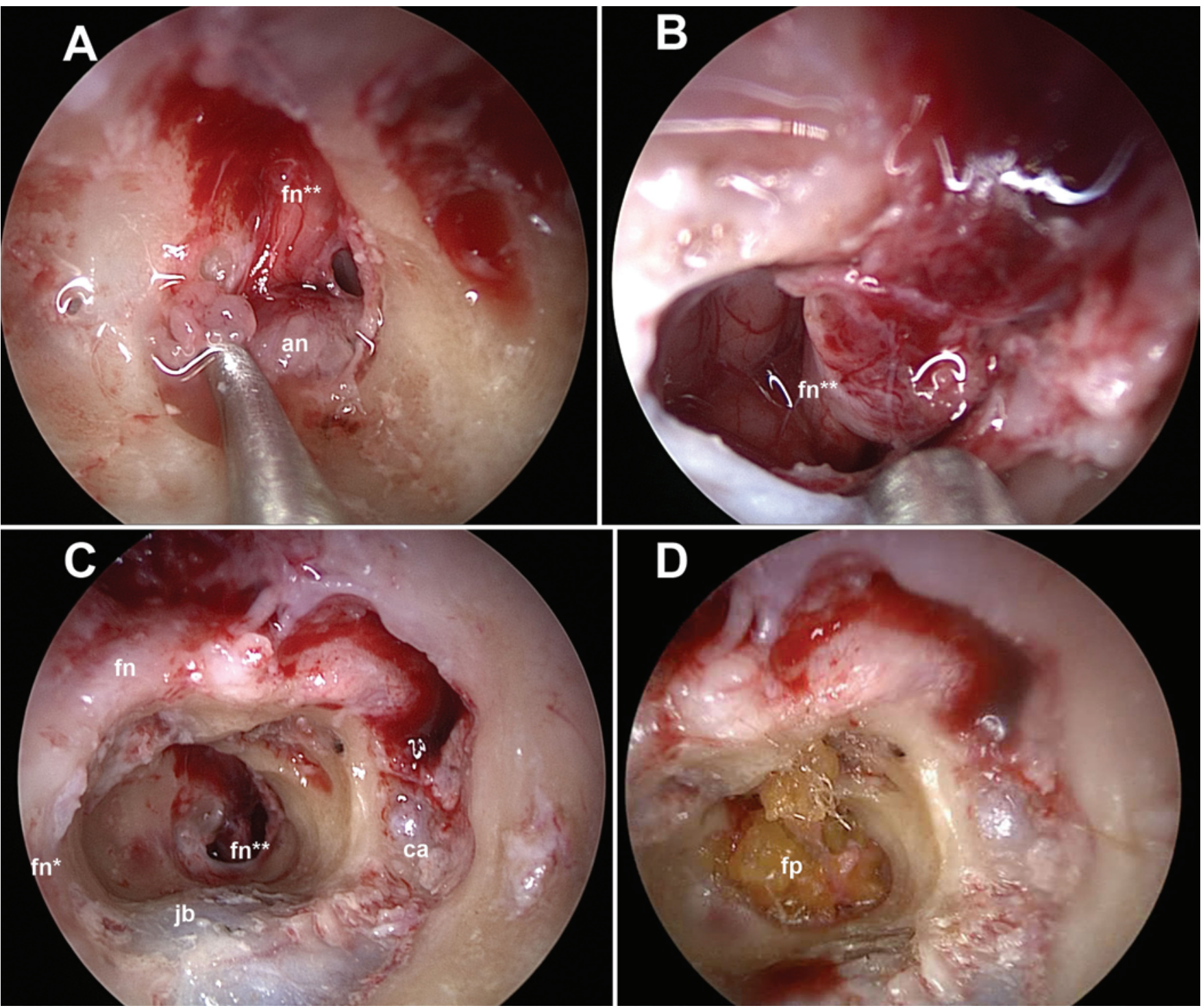

FIG. 5. Photographs of EETTA in the right ear. A: The VS was removed after facial nerve detection in the IAC. B: Endoscopic check of the facial nerve integrity from the IAC to the CPA. C: Final tympanic cavity after VS removal. D: A fat pad was used to close the promontorial defect. Figure is available in color online only.

growing mass over time. When mass growth has been documented, a specific therapeutic approach can be more strongly suggested.

Therapy for patients with VS includes radiation (mainly radiosurgery) or surgery. The medical literature has already documented good control of VS with radiosurgery, ${ }^{3}$ but studies that prospectively compare radiosurgery to a wait-and-scan strategy are lacking. Therefore, in the present series we do not know exactly which part of the mass growth was stopped by radiosurgery and what percentage would have stopped as a natural course, as often occurs. Moreover, long-term results of radiosurgery are less available and are possibly influenced by a decrease in sample size and lack of censorization. In young patients, the choice of radiosurgery today cannot exclude possible reoperation tomorrow, with a higher risk of complications.

We present the first case series of patients who under- went surgery via the EETTA for the treatment of VS. In 2013, our team documented the first EETTA. ${ }^{8}$ The approach guaranteed the removal of CS with IAC extension. After this first clinical application, we started using the approach more frequently in patients with Koos Grade I or II VS. The EETTA offers lateral-to-medial control of the IAC, providing high magnification of every structure inside and outside the IAC, including the facial nerve. According to our initial results, the mobility was comparable to that of a tympanoplasty rather than an operation of the CPA. The sample size in this study is small because we very carefully selected patients for the surgery. At present, we would summarize the indications for an EETTA in the treatment of VS to be as follows: 1) a growing, Koos Grade I or II VS; 2) AAO-HNS Class D hearing status; and 3) symptoms that do not respond to medical treatment (e.g., intratympanic gentamicin injections in the case of 
TABLE 1. Summary of clinical data in 10 patients who underwent surgery via the EETTA for VA in the IAC

\begin{tabular}{|c|c|c|c|c|c|c|c|c|c|}
\hline \multirow[b]{2}{*}{$\begin{array}{l}\text { Case } \\
\text { No. }\end{array}$} & \multirow[b]{2}{*}{$\begin{array}{l}\text { Age } \\
\text { (yrs) }\end{array}$} & \multirow[b]{2}{*}{ Sex } & \multirow[b]{2}{*}{ Disease } & \multicolumn{2}{|c|}{ RGS Grade } & \multirow[b]{2}{*}{$\begin{array}{l}\text { Tumor Size: } \\
\text { Koos Grade }\end{array}$} & \multirow[b]{2}{*}{$\mathrm{FU}$ (mos) } & \multirow[b]{2}{*}{ Postop Complication } & \multirow[b]{2}{*}{$\begin{array}{l}\text { Hospital } \\
\text { Stay (days) }\end{array}$} \\
\hline & & & & $\begin{array}{l}\text { Preop Facial } \\
\text { Function }\end{array}$ & $\begin{array}{c}\text { Facial Function } \\
\text { at Last FU }\end{array}$ & & & & \\
\hline 1 & 44 & M & VS & 1 & 1 & I & 12 & None & 6 \\
\hline 2 & 40 & M & CS & 1 & 1 & I & 37 & Granulation tissue in EAC & 10 \\
\hline 3 & 62 & $\mathrm{~F}$ & VS & 1 & 1 & I & 21 & Temporary facial palsy & 5 \\
\hline 4 & 45 & M & Meningioma & 1 & 1 & I & 12 & None & 7 \\
\hline 5 & 65 & $\mathrm{~F}$ & VS & 1 & 1 & I & 5 & None & 6 \\
\hline 6 & 40 & $\mathrm{~F}$ & VS & 1 & $\|/\| \|$ & II & 3 & Facial palsy; RGS Grade II/III & 6 \\
\hline 7 & 50 & M & VS & 1 & II & II & 2 & Facial palsy, RGS Grade II & 9 \\
\hline 8 & 27 & $\mathrm{~F}$ & VS & 1 & 1 & I & 4 & None & 8 \\
\hline 9 & 66 & $\mathrm{~F}$ & VS & 1 & III/IV & I & 4 & Facial palsy, RGS Grade III/IV & 8 \\
\hline 10 & 53 & $\mathrm{~F}$ & VS & 1 & 1 & II & 1 & None & 6 \\
\hline
\end{tabular}

FU $=$ follow-up.

debilitating vertigo). Despite these very strict indications, we assert that EETTA is a very promising technique since it differs from the classic microscopic approaches in terms of mobility. Moreover, it guarantees radical removal of the pathology with very low mobility to the facial nerve because of the direct control and magnification of the whole nerve path provided by the endoscope. Our good results are possibly attributable to the early stage of the VS and to the loose adhesions of the mass to the nerve. Of course, hearing preservation is not feasible with this approach; therefore, we recommend an EETTA only for patients with unserviceable hearing. It should be noted that most patients who underwent an EETTA had VS within the fundus of the IAC, so hearing preservation would have been unlikely with any other approach as well (e.g., retrosigmoid).

It must also be noted that in the present series, none of the patients required an ICU stay, and they were directly extubated and sent to the inpatient clinic after surgery, just as they would with any middle ear surgery. In addition, the patients' hospital stay was much less than 1 week, although it was prudentially longer for the first $2-3$ patients. Compared with the time required for traditional approaches, operative time for the EETTA was markedly reduced (roughly 3 hours), and an even further shortening of surgical times can be imagined in the future.

The EETTA prevents the possibility of a cochlear implant on the same side, but the use of cochlear implants for single-sided deafness is highly controversial. In most patients, any kind of prosthesis (whether conventional or implantable) cannot modify quality of life by an amount that justifies the expense. Moreover, the chances of cochlear nerve preservation with VS surgery are low overall, and the nerve remains viable in only a few patients. Anatomical preservation does not imply useful hearing postoperatively, so patients may not benefit from a prosthesis. This is particularly true for patients with cochlear implants, in whom only initial experiences in single-sided deafness have been reported in the literature and for whom no clinical trials comparing results between a study group and a control group are available.

\section{Conclusions}

The EETTA proved to be successful for the removal of VS or meningioma involving the cochlea, fundus, and IAC, with possibly lower complication rates and less invasive procedures than in traditional microscopic approaches. The potential for the extensive and routine application of this approach in lateral and posterior skull base surgery will depend on the development of technology and surgical refinements, as well as on the diffusion of skull base endoscopic skills within the otolaryngological and neurosurgical communities.

\section{References}

1. Alicandri-Ciufelli M, Piccinini A, Grammatica A, Salafia F, Ciancimino C, Cunsolo E, et al: A step backward: the 'Rough' facial nerve grading system. J Craniomaxillofac Surg 41:e175-e179, 2013

2. Bennett M, Haynes DS: Surgical approaches and complications in the removal of vestibular schwannomas. Otolaryngol Clin North Am 40:589-609, ix-x, 2007

3. Jacob JT, Pollock BE, Carlson ML, Driscoll CL, Link MJ: Stereotactic radiosurgery in the management of vestibular schwannoma and glomus jugulare: indications, techniques, and results. Otolaryngol Clin North Am 48:515-526, 2015

4. Magnan J, Chays A, Lepetre C, Pencroffi E, Locatelli P: Surgical perspectives of endoscopy of the cerebellopontine angle. Am J Otol 15:366-370, 1994

5. Marchioni D, Alicandri-Ciufelli M, Mattioli F, Nogeira JF, Tarabichi M, Villari D, et al: From external to internal auditory canal: surgical anatomy by an exclusive endoscopic approach. Eur Arch Otorhinolaryngol 270:1267-1275, 2013

6. Marchioni D, Alicandri-Ciufelli M, Molteni G, Genovese E, Presutti L: Endoscopic tympanoplasty in patients with attic retraction pockets. Laryngoscope 120:1847-1855, 2010

7. Marchioni D, Alicandri-Ciufelli M, Rubini A, Presutti L: Endoscopic transcanal corridors to the lateral skull base: Initial experiences. Laryngoscope 125 (Suppl 5):S1-S13, 2015

8. Presutti L, Alicandri-Ciufelli M, Cigarini E, Marchioni D: Cochlear schwannoma removed through the external auditory canal by a transcanal exclusive endoscopic technique. Laryngoscope 123:2862-2867, 2013

9. Presutti L, Marchioni D, Mattioli F, Villari D, AlicandriCiufelli M: Endoscopic management of acquired choles- 
teatoma: our experience. J Otolaryngol Head Neck Surg 37:481-487, 2008

10. Presutti L, Nogueira JF, Alicandri-Ciufelli M, Marchioni D: Beyond the middle ear: endoscopic surgical anatomy and approaches to inner ear and lateral skull base. Otolaryngol Clin North Am 46:189-200, 2013

11. Tarabichi M: Endoscopic management of limited attic cholesteatoma. Laryngoscope 114:1157-1162, 2004

12. Thakur JD, Banerjee AD, Khan IS, Sonig A, Shorter CD, Gardner GL, et al: An update on unilateral sporadic small vestibular schwannoma. Neurosurg Focus 33(3):E1, 2012

13. Thomassin JM, Korchia D, Doris JM: Endoscopic-guided otosurgery in the prevention of residual cholesteatomas. Laryngoscope 103:939-943, 1993

\section{Disclosures}

The authors report no conflict of interest concerning the materials or methods used in this study or the findings specified in this paper.

\section{Author Contributions}

Conception and design: Marchioni, Pavesi, Presutti. Acquisition of data: Marchioni, Rubini, Masotto. Analysis and interpretation of data: Alicandri-Ciufelli, Marchioni. Drafting the article: Alicandri-Ciufelli, Marchioni, Rubini, Pavesi. Critically revising the article: Presutti. Reviewed submitted version of manuscript: Alicandri-Ciufelli. Approved the final version of the manuscript on behalf of all authors: Alicandri-Ciufelli. Administrative/techni$\mathrm{cal} /$ material support: Rubini, Masotto, Presutti. Study supervision: Presutti.

\section{Supplemental Information}

Videos

Video 1. https://vimeo.com/149286474.

\section{Correspondence}

Matteo Alicandri-Ciufelli, Department of Otolaryngology, University Hospital of Modena, Via del Pozzo 71, Modena 41100, Italy. email: matteo.alicandri@hotmail.it. 